\title{
Coulomb Barrier to Tunneling between Parallel Two-Dimensional Electron Systems
}

\author{
J. P. Eisenstein, L. N. Pfeiffer, and K. W. West \\ AT\&T Bell Laboratories, Murray Hill, New Jersey 07974
}

(Received 23 October 1992)

\begin{abstract}
Tunneling between parallel two-dimensional electron gases in double quantum wells is examined at both zero and high perpendicular magnetic field. The measured $I-V$ characteristics show the magnetic field qualitatively alters the tunneling density of states, creating both a wide gap at the Fermi level and a much broader density of final states than is present at zero field. We suggest the origin of these effects lies in the strong Coulomb correlations characteristic of Landau quantized two-dimensional systems.
\end{abstract}

PACS numbers: 73.20.Dx, 73.40.Gk

Application of a large enough magnetic field to a twodimensional electron gas (2DEG) forces all the electrons into the lowest Landau level. In this extreme quantum limit the kinetic energy is quenched and Coulomb correlations completely dominate the collective dynamics. The quantum liquid states associated with the fractional quantum Hall effect (FQHE) [1], the mounting evidence [2] for Wigner crystallization, and the recent interest [3] in the nature of the electron gas at half filling of the lowest Landau level, all derive from the strongly correlated nature of the 2DEG at high field. By now many standard techniques have been applied to this system, including transport, optics, ultrasonics, capacitance, etc. Tunneling into the 2DEG at high fields, however, has received relatively little attention [4,5].

Here we report on our studies of tunneling between two parallel 2DEGs separated by a semiconductor barrier. While the zero field results are explicable using a simple single-particle picture of the electron gas, the high magnetic field data are clearly not. Two aspects of the high field results stand out: First, a wide tunneling gap, centered at the Fermi level, is found over a broad range of magnetic field and Landau level filling fraction; and second, the density of states into which electrons may tunnel above this gap is much broader than the zero field tunnel resonance. We attribute these effects to the strongly correlated nature of the electron systems and argue that disorder plays only a subsidiary role. Although departures from the single-particle picture are evident for fields as low as $B \sim 0.5 \mathrm{~T}$, we concentrate here on the extreme quantum limit where only the lowest Landau level is occupied.

For these experiments we employ high mobility bilayer 2D electron systems in double quantum wells (DQW) fabricated by molecular beam epitaxy. Each 2D gas occupies the lowest subband of a 200 - $\AA$-wide GaAs quantum well embedded in $\mathrm{Al}_{0.3} \mathrm{Ga}_{0.7} \mathrm{As}$. The barrier separating the two wells is $175 \AA$ wide and roughly $250 \mathrm{meV}$ high. Si $\delta$-doping layers deposited above and below the DQW create two nearly equivalent 2DEGs with sheet densities $n_{1,2} \approx 1.6 \times 10^{11} \mathrm{~cm}^{-2}$ and low-temperature mobilities near $3 \times 10^{16} \mathrm{~cm}^{2} / \mathrm{Vs}$. The sample is patterned into a square mesa $250 \mu \mathrm{m}$ on a side, to which four narrow arms are attached. Separate Ohmic contacts to the individual layers are established at the ends of these arms using a selective depletion technique [6]. These contacts allow not only magnetotransport measurements on the individual layers, but also the application of a voltage $V$ directly between the two 2D systems and the measurement of the resulting tunneling current. The currentvoltage $(I$ vs $V)$ and conductance-voltage $(d I / d V$ vs $V)$ characteristics presented here are two-terminal. This is not a problem at $B=0$ since the typical tunneling resistance $(\sim 1 M \Omega)$ greatly exceeds all other relevant resistances. As discussed below, however, the reduced conductivity $\sigma_{x x}$ at high $B$, especially as strong quantum Hall states, can restrict the field and temperature ranges studied. As grown, the densities of the two 2D layers typically differ by $(5-10) \%$. This imbalance can be canceled out by applying a small voltage to an overall back-side gate electrode. For almost all the results discussed in this paper the DQW has been balanced and $n_{1}=n_{2}$. This density balance is disrupted, however, when the interlayer tunneling voltage $V$ is applied, due to the capacitance between the 2D sheets. Low field magnetotransport measurements have shown $n_{j}(V) / n_{j}(0)=1 \pm V / 90$, with $V$ in $\mathrm{mV}$; the sign is opposite for the two layers.

Figure 1 displays the central qualitative result of this paper. The low-temperature tunneling conductance $d I /$ $d V$ vs $V$ is displayed at both zero magnetic field and $B=13 \mathrm{~T}$ where the lowest Landau level in each 2DEG is roughly half filled. At zero field a sharp resonance is observed while the high data exhibit a region of nearly zero conductance centered at $V=0$, flanked by broad ranges of voltage over which substantial tunneling exists. Measurements of the zero-bias tunneling conductance at $B=13 \mathrm{~T}$ and $T=50 \mathrm{mK}$ show that it is at least 4000 times smaller than the peak tunneling conductance observed at $B=0$. We stress that the resistivity of the individual 2DEGs does not reflect this high field tunneling gap, showing instead the familiar features of the integral and fractional quantum Hall effects.

The zero field data in Fig. 1 are consistent with a simple single-particle picture [7] of the tunneling process. Conservation of energy and in-plane momentum forbids purely $2 \mathrm{D}-2 \mathrm{D}$ tunneling unless the subband edges of the two systems are closely aligned, $\Delta E_{0} \approx 0$. Thus the voltage location of the resonance is just $V=\left(E_{F, 1}-E_{F, 2}\right) / e$ 


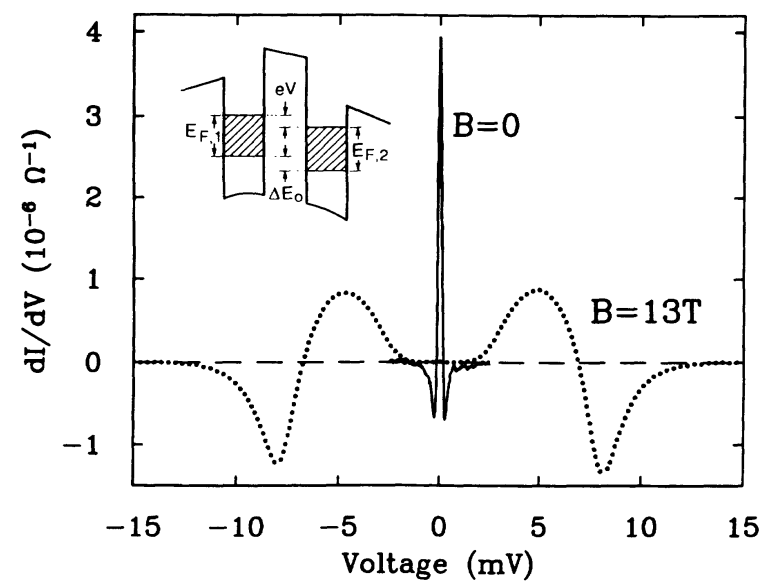

FIG. 1. Low-temperature tunneling conductance $d I / d V$ vs interlayer voltage at zero magnetic field $(T=0.3 \mathrm{~K})$ and $B=13$ $\mathrm{T}(T=0.66 \mathrm{~K})$ where the lowest Landau level is approximately half filled. A simplified band diagram, appropriate to $B=0$, defines relevant energies.

with $E_{F, j}$ the Fermi energies of the two 2DEGs. Changing the density in the lower layer, via the back gate, shifts the resonance in accordance with this formula. The balance condition $n_{1}=n_{2}$ (implying $E_{F, 1}=E_{F, 2}$ ) is obtained by centering the $B=0$ resonance at $V=0$, as in Fig. 1 . The observed resonance width, $\Gamma_{0} \approx 0.3 \mathrm{meV}$, is much less than the Fermi energy of the 2D gases, $E_{F}=5.7$ $\mathrm{meV}$, reflecting a high degree of momentum conservation. This linewidth is consistent with an estimate of the electronic lifetime based upon the minimum magnetic field required to resolve Shubnikov-de Haas oscillations in the resistivity $\rho_{x x}$. We note, however, that various inhomogeneous broadening mechanisms, e.g., quantum well width fluctuations, may also contribute to the observed linewidth.

Returning to high magnetic fields, Fig. 2 shows a series of $I-V$ characteristics taken at fields from $B=8 \mathrm{~T}$ to almost $14 \mathrm{~T}$. Only data for positive $V$ are shown, the $I-V$ data being antisymmetric about $V=0$. These data, taken at $T=0.6 \mathrm{~K}$, reveal additional important results. The basic features of the tunneling below about $10 \mathrm{meV}$ (to which only the lowest Landau level of each 2DEG contributes) show only subtle changes with magnetic field even though the Landau level filling fraction $v=h n / e B$ changes from $v=0.83$ to 0.48 . Above the gap around $V=0$ the tunneling current shows a broad intra-Landau level peak before collapsing into the inter-Landau level gap. The arrows indicate the onset energy $2 \Delta_{1}$ for tunneling above the Fermi level gap, and $2 \Delta_{2}$, the energy at which the peak current is reached. (The onset is defined as the energy at which the current attains $1 \%$ of the peak value.) The field dependences of $2 \Delta_{1,2}$ are shown in the inset to the figure. As the solid line inset shows, these energies are comparable to the Coulomb energy $E_{C}$ $=e^{2} / \varepsilon\langle a\rangle$, with the interelectron spacing $\langle a\rangle=2(\pi n)^{-1 / 2}$.

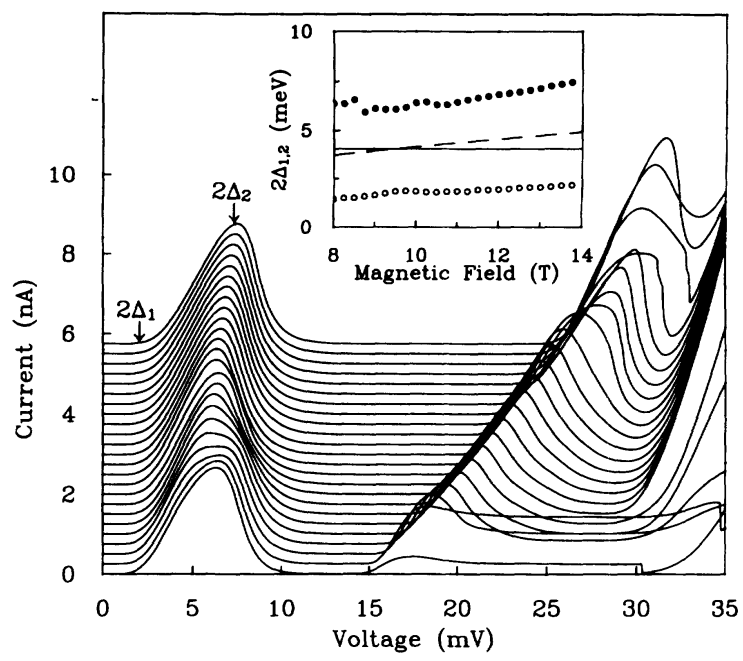

FIG. 2. Tunneling $I-V$ characteristics at $T=0.6 \mathrm{~K}$ for magnetic fields from $B=8 \mathrm{~T}$ to $13.75 \mathrm{~T}$ in steps of $0.25 \mathrm{~T}$. The curves are vertically offset for clarity. Inset: Magnetic field dependence of the onset $\left(2 \Delta_{1}\right)$ and peak $\left(2 \Delta_{2}\right)$ voltages. The solid line is the Coulomb energy $E_{C}=e^{2} / \varepsilon\langle a\rangle$ while the dashed line is $0.3 e^{2} / \varepsilon l_{0}$.

At higher energy a second peak in the $I-V$ is seen, whose position shifts upward linearly with magnetic field. We identify this second peak with tunneling into the first excited Landau level. The energy of this inter-Landau level transition is systematically larger than the bare cyclotron energy $\hbar \omega_{c}$ by about $30 \%$, an enhancement consistent with recent estimates of Coulomb effects [8]. At the highest voltages the current rises again, signaling the onset of tunneling into the first excited subband of the quantum well.

Tunneling data from the upper spin branch of the lowest Landau level $(2>v>1,6.6>B>3.3 \mathrm{~T})$ also show a pronounced gap around $V=0$ but, as greater structure is found above the gap than is seen in the $1>v$ case, we defer its discussion to a future publication. Preliminary data have also been obtained for the case where $n_{1} \neq n_{2}$ by using the back gate to change the lower layer density $n_{2}$. A $25 \%$ decrease in $n_{2}$ produces a negligible shift of the gap center away from $V=0$, but the gap width (as measured by $2 \Delta_{1}$ ) decreases by an amount comparable to the change in the Coulomb energy $E_{C}$.

Figure 3 summarizes the temperature dependence of the high field tunneling conductance. The upper panel shows that the gap is almost completely filled in at $T=9.5 \mathrm{~K}$, while the lower panel reveals that the zero-bias conductance, $d I / d V$ at $V=0$, exhibits a thermally activated regime. This has been found throughout the lowest Landau level. The inset displays the observed activation energy $E_{A}$ versus magnetic field in both spin branches of the lowest Landau level. As expected, from analogy to superconducting tunnel junctions [9], $E_{A}$ is roughly one-half the onset energy $2 \Delta_{1}$. For $2>v>1 E_{A}$ 

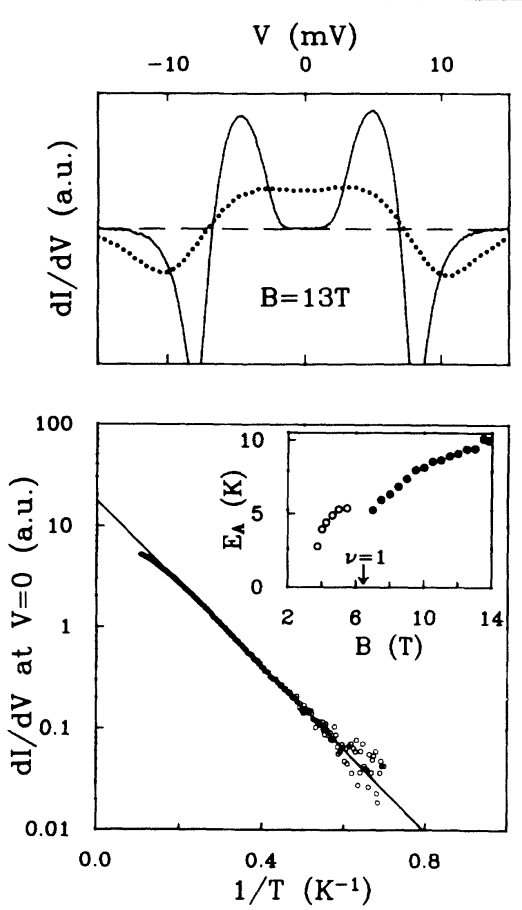

FIG. 3. Upper panel: Tunneling conductance at $B=13 \mathrm{~T}$ for $T=0.66 \mathrm{~K}$ (solid) and $T=9.5 \mathrm{~K}$ (dotted). Lower panel: Zero-bias conductance vs $1 / T$ at $B=13 \mathrm{~T}$ showing activated behavior. Inset: Activation energy vs field. Solid dots, $v<1$; open dots, $2>v>1$.

rises quite rapidly above about $3.75 \mathrm{~T}$ where $E_{A}=2.8 \mathrm{~K}$. At this field $E_{A}$ is comparable to the disorder broadening $\Gamma_{0} \sim 3.5 \mathrm{~K}$. This may reflect a boundary between a low field regime where disorder is important and a high field range where its role is secondary.

Note that no data are shown near $v=1$ at $B=6.6 \mathrm{~T}$. The integer quantum Hall effect at this field, and the associated extremely small values of $\sigma_{x x}$ at low temperature, restrict the in-plane current flow to a boundary region at the mesa edge. We find the tunneling current in this situation is strongly suppressed. This supports our conclusion [10] that the tunneling is fairly uniform across the mesa area provided $\sigma_{x x}$ is not unduly small. The data in Fig. 2 were taken at $T=0.6 \mathrm{~K}$ to avoid similar problems near the $v=\frac{2}{3}$ FQHE state at $B=9.9 \mathrm{~T}$. Even so, the measured value of $\sigma_{x x}$ falls by a factor of 3 on going from $B=8$ to $14 \mathrm{~T}$ (due largely to the rising Hall resistance). The only subtle variations observed in the (intra-Landau level) tunneling current also suggest $\sigma_{x x}$ has little impact.

The primary effects of the magnetic field, the broad gap around $V=0$, and the wide band of higher energies into which tunneling may proceed, are inconsistent with a simple noninteracting electron picture. With partially filled Landau levels in both layers a nonzero tunneling conductance $d I / d V$ would then be expected at $V=0$. The width of the resonance would, in analogy to the zero field case, reflect the disorder broadening $\Gamma_{B}$ of the Landau levels. While $\Gamma_{B}$ may exceed the width $\Gamma_{0}=0.3 \mathrm{meV}$ seen at zero field, it is highly unlikely that it is comparable to the several meV range $\left(2 \Delta_{2}-2 \Delta_{1} \sim 5 \mathrm{meV}\right)$ over which substantial tunneling is observed at high $B$. Indeed, the clear observations of the FQHE at $v=\frac{1}{3}$ and $\frac{2}{3}$, in both transport and a thermodynamic compressibility study (on this and other samples taken from the same molecular beam epitaxy wafer), demonstrate that electron-electron interaction effects dominate over disorder [11].

A possible explanation of these tunneling anomalies lies in the strongly correlated nature of a $2 \mathrm{DEG}$ throughout the lowest Landau level. The high field localizes electrons on the scale of the magnetic length $l_{0}$ allowing them to avoid one another much more effectively than at $B=0$. At large enough field Wigner crystallization results [2]. While the present studies are in the electron liquid regime, the 2DEGs remain strongly correlated, and at short distances resemble the crystalline state. An electron tunneling between two such correlated liquids must first be extracted from one and then injected into the other. Assuming a given tunneling event occurs instantaneously [12], the minimum energy required to inject an electron into an "interstitial" position in the liquid will be of order $E_{C}=e^{2} / \varepsilon\langle a\rangle$. As time evolves, this "defect" is relaxed and the liquid ground state of the $(N+1)$-particle system is restored. A similar energy cost attends the "vacancy" left behind by the extraction of an electron. These energy penalties suppress tunneling at the Fermi level, creating a pseudogap. As the inset to Fig. 2 demonstrates, the observed tunneling gap is comparable to $E_{C}$. The localized nature of these defects implies that a wide range of magnetophonon modes are emitted in the relaxation process and this, coupled with the likelihood of multiphonon processes, is likely the source of the broad tunneling peaks flanking the gap. In this model the gap is a single layer correlation effect; for these bilayer systems the $I-V$ characteristics would represent a convolution of the gaps in the two layers.

The fractional quantum Hall effect is ignored in this picture. Our view is that the FQHE, though creating a true, thermodynamic energy gap, represents a relatively subtle additional correlation in the system. Both the $v=\frac{2}{3}$ and $\frac{3}{5}$ FQHE states, at $B=9.9$ and $11 \mathrm{~T}$, respectively, are easily seen in ordinary transport but, as Fig. 2 proves, they have little impact on the $I-V$ curves. (The data do suggest an unresolved splitting of the tunneling peak at $V \sim 7 \mathrm{mV}$ around $9 \mathrm{~T}$ which may be related to the $\frac{2}{3}$ state.) Equally ignored are excitonic effects. As the quantum well separation is comparable to the distance $\langle a\rangle$ between electrons in each layer, this may not be a very good approximation. Future experiments with different layer spacings should help clarify this.

It is well known that the interplay of Coulomb interactions with disorder can produce singular tunneling gaps at the Fermi level. In 2D logarithmic gaps were predicted by 
Altshuler, Aronov, and Lee [13] in the diffusive regime while Efros and Shklovskii [14] found linear Coulomb gaps in the Mott hopping case. By contrast, our results are in the clean limit. The gap we find, while centered at the Fermi level, is not singular there. We believe it would exist in a disorder-free 2D gas. Recently Efros [15] has presented numerical evidence for a Coulomb gap in a clean classical electron system with quenched kinetic energy at temperatures high enough to melt the Wigner solid. This may be relevant here since high magnetic fields make real 2D systems increasingly "classical."

Finally, we remark that suppression of tunneling into a 2D system at high magnetic fields has been previously reported by Ashoori et al. [5]. While no $I-V$ characteristics were obtained, the zero-bias tunneling conductance between the 2D gas and a 3D electron system was measured. The suppression observed at high field did not exhibit the thermally activated regime we find. Based upon the temperature dependence, Ashoori et al. concluded that a singular gap existed at the Fermi level and good fits using linear gaps were obtained. This contrasts sharply with the broad energy gap that we observe. A basic difference between our samples and theirs is the degree of disorder. Although not measured, Ashoori et al. estimated the 2D mobility to be about $10^{5} \mathrm{~cm}^{2} / \mathrm{Vs}$, some 30 times smaller than for the present samples. While we speculate our high field results derive from a pure $2 \mathrm{D}$ system it seems plausible that theirs depend critically upon the interplay of disorder and interaction. Whether a smooth connection (as a function of disorder, say) exists between these two results remains unclear.

In summary, we have found that high magnetic fields dramatically alter the tunneling between two high purity 2D electron gases. A broad Fermi level tunneling gap is observed over wide ranges of magnetic field. We have proposed an explanation based upon the highly correlated nature of the 2D electron gas in the lowest Landau level, and not upon the presence of disorder.

It is a pleasure to thank B. I. Halperin, S. He, R. B. Laughlin, P. B. Littlewood, A. H. MacDonald, D. Monroe, A. Pasquarello, P. M. Platzman, C. M. Varma, and especially M. S. Hybertsen for many useful discussions.

[1] For a review of the FQHE, see The Quantum Hall Effect, edited by R. E. Prange and S. M. Girvin (SpringerVerlag, New York, 1987); T. Chakraborty and P. Pietilainen, The Fractional Quantum Hall Effect, Springer Series in Solid State Sciences Vol. 85 (Springer-Verlag, Berlin, 1988).

[2] For a recent example, see M. A. Paalanen et al., Phys. Rev. B 45, 11342 (1992), and references cited therein.

[3] R. L. Willett et al., Phys. Rev. Lett. 65, 112 (1990); B. I. Halperin, P. A. Lee, and N. Read (to be published).

[4] J. Smoliner, E. Gornik, and G. Weimann, Appl. Phys. Lett. 52, 2136 (1988).

[5] R. C. Ashoori, J. A. Lebens, N. P. Bigelow, and R. H. Silsbee, Phys. Rev. Lett. 64, 681 (1990).

[6] J. P. Eisenstein, L. N. Pfeiffer, and K. W. West, Appl. Phys. Lett. 57, 2324 (1990).

[7] J. P. Eisenstein, L. N. Pfeiffer, and K. W. West, Appl. Phys. Lett. 58, 1497 (1991).

[8] A. P. Smith, A. H. MacDonald, and G. Gumbs, Phys. Rev. B 45, 8829 (1992).

[9] See Principles of Electron Tunneling Spectroscopy, by E. L. Wolf (Oxford Univ. Press, New York, 1985).

[10] Based in part on numerical simulations of the current distribution.

[11] J. P. Eisenstein, L. N. Pfeiffer, and K. W. West, Phys. Rev. Lett. 68, 674 (1992). Based on this work we estimate $\Gamma_{B} \sim 0.6 \mathrm{meV}$ at $B=12 \mathrm{~T}$ [J. P. Eisenstein (unpublished)].

[12] We estimate the tunneling time from the electron's (imaginary) velocity under the barrier [M. Buttiker and R. Landauer, Phys. Rev. Lett. 49, 1739 (1982)] to be $\tau_{T} \sim 15 \mathrm{fs}$. This is much less than the period of zoneboundary magnetophonons in the Wigner crystal [R. Cote and A. H. MacDonald, Phys. Rev. Lett. 65, 2662 (1990)]; the liquid state would presumably relax even slower.

[13] B. L. Altshuler, A. G. Aronov, and P. A. Lee, Phys. Rev. Lett. 44, 1288 (1980).

[14] A. L. Efros and B. I. Shklovskii, in Electron-Electron Interactions in Disordered Systems, edited by A. L. Efros and M. Pollak (Elsevier, Amsterdam, 1985), p. 409.

[15] A. L. Efros, Phys. Rev. Lett. 68, 2208 (1992). 